\title{
Diagnosis of lateral pelvic lymph node metastasis of T1 lower rectal cancer using diffusion-weighted magnetic resonance imaging: A case report with lateral pelvic lymph node dissection of lower rectal cancer
}

\author{
SHIMPEI OGAWA, MICHIO ITABASHI, TOMOICHIRO HIROSAWA, \\ TAKUZO HASHIMOTO, YOSHIKO BAMBA and TAKAHIRO OKAMOTO
}

Department of Surgery II, School of Medicine, Tokyo Women's Medical University, Shinjuku-ku, Tokyo 162-8666, Japan

Received December 11, 2015; Accepted February 25, 2016

DOI: $10.3892 / \mathrm{mco} .2016 .797$

\begin{abstract}
The present study presented a 35-year-old female patient in whom fecal occult blood was detected in a medical check-up. Colonoscopy revealed a superficial elevated-type tumor with central depression in the lower rectum. The tumor was diagnosed as T1 deep invasive cancer. No swollen lymph nodes or distant metastasis were found on computed tomography or $\left[{ }^{18} \mathrm{~F}\right]$-fluorodeoxyglucose-positron emission tomography with computed tomography. However, a swollen right lateral pelvic lymph node (LPLN; short axis $4 \mathrm{~mm}$ ) was revealed on magnetic resonance imaging (MRI). This lymph node exhibited high intensity on diffusion-weighted imaging (DWI), suggesting metastasis. Low anterior resection, regional lymph node dissection and right LPLN dissection (LPLD) were performed. Histological analysis revealed metastasis in the right LPLN, as suggested by the high DWI intensity. The indication for LPLD in the current Japanese guidelines is based on the tumor location and depth of invasion ( $\geq \mathrm{T} 3$ ), however, not on the status of LPLN metastasis in pre-operative evaluation. The present case was cT1, which is not included in this indication. DWI is sensitive for the diagnosis of lymph node metastasis of colorectal cancer, although inflammation-induced swelling of lymph nodes in advanced rectal cancer may cause a false-positive result, which is uncommon in T1 cases. Therefore, an LPLN with a high intensity DWI signal in T1 cases is likely to be metastasis-positive. Pre-operative DWI-MRI may be useful for identifying LPLN metastasis when planning the
\end{abstract}

Correspondence to: Dr Michio Itabashi, Department of Surgery II, School of Medicine, Tokyo Women's Medical University, 8-1 Kawada-cho, Shinjuku-ku, Tokyo 162-8666, Japan

E-mail: itabashi@surg2.twmu.ac.jp

Key words: lateral pelvic lymph node, lateral pelvic lymph node dissection, T1 lower rectal cancer, diffusion-weighted imaging, magnetic resonance imaging treatment strategy in these cases. The present study suggested reinvestigation of the indication for LPLD with inclusion of LPLN status on pre-operative imaging.

\section{Introduction}

Strategies for the treatment of lateral pelvic lymph node (LPLN) metastasis in patients with lower rectal cancer has been controversial. In Western countries (1,2), LPLN dissection (LPLD) is rarely used since LPLN metastasis is viewed as a systemic disease. By contrast, in Japan, LPLN is categorized according to the regional lymph node and LPLN metastasis is treated as a localized lesion (3). Notably, a previous Japanese nationwide multi-institutional study (4) demonstrated that the survival rate of patients with internal iliac lymph node metastasis was comparable to that of cases with a tumor, node, metastasis (TNM) classification of N2a, and that the survival rate of patients with LPLN metastasis, which is more distant than the internal iliac lymph node, was comparable to that in cases with a classification of $\mathrm{N} 2 \mathrm{~b}$. These previous findings suggested that LPLN metastasis can be recognized as local disease. The 5 year overall and cancer-specific survival rates of patients with LPLN metastasis were better compared with those of stage IV patients following curative resection (4). Total mesorectal excision (TME) with LPLD is the established method for advanced lower rectal cancer, and LPLD in Japan was effective in reducing the intrapelvic recurrence by $50 \%$ and improving the 5 year survival rate by $8-9 \%$ (5).

The diagnostic utility of diffusion-weighted imaging (DWI) in magnetic resonance imaging (MRI) for patients with colorectal cancer has previously been reported $(6,7)$. DWI detects the random movement of water molecules in tissue. This diffusion is inhibited in densely proliferating cells, including fibrosis, edema and tumors, and this is visualized as an abnormally high intensity signal (8). Furthermore, diffusion capacity decreases in malignant tumors compared with that in benign tumors as a result of the higher cell density, and therefore, DWI can differentiate between malignant and benign tumor types $(8,9)$. The signal intensities of primary lesions and lymph node metastases on DWI are also higher compared with 

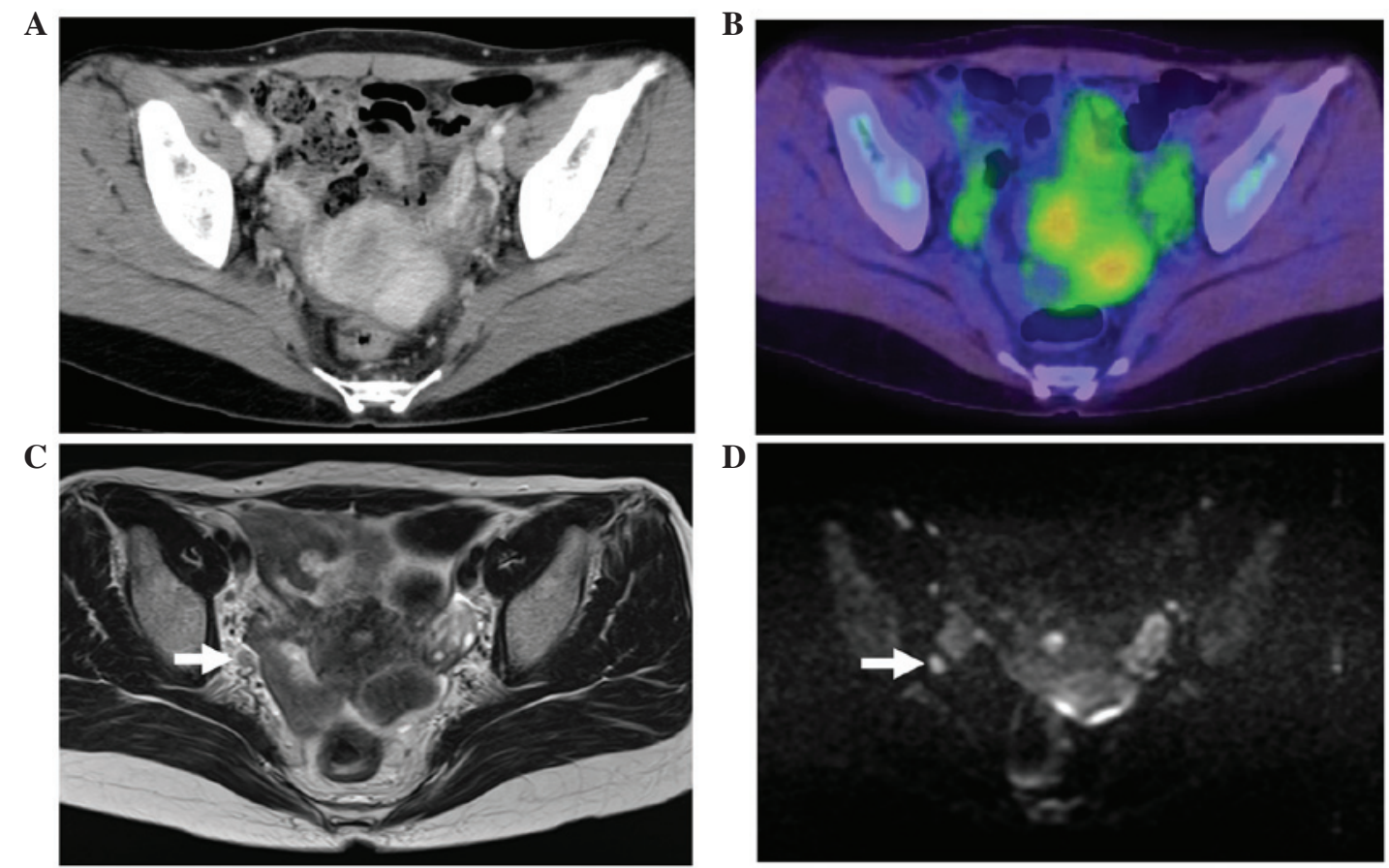

Figure 1. (A) CT revealed no swollen lymph node in the right lateral region. (B) Similarly, $\left[{ }^{18} \mathrm{~F}\right]$-fluorodeoxyglucose-positron emission tomography/CT revealed no abnormal accumulation in the right lateral region, indicating the absence of lymph node metastasis. (C) T2-weighted magnetic resonance imaging revealed a swollen lymph node with a short axis of $4 \mathrm{~mm}$ in the right lateral region (arrow). (D) This lymph node exhibited a high intensity on diffusion-weighted imaging, suggesting lymph node metastasis (arrow). CT, computed tomography.

that of the surrounding area, and this high contrast facilitates the detection of lesions.

In a multicenter cooperative study of 1,427 patients with lower rectal cancer in Japan, the incidences of LPLN metastasis was $16.7 \%$ in cases treated with LPLD and $9.8 \%$ in all patients including without LPLD, respectively (10). By depth of tumor invasion, the incidences were $0.9,5.4,13.5$ and $28.8 \%$ in $\mathrm{T} 1$, $\mathrm{T} 2, \mathrm{~T} 3$ and $\mathrm{T} 4$ cases, respectively (10).

A patient with T1 lower rectal cancer with LPLN metastasis, which is relatively uncommon, in whom diagnosis by DWI MRI was useful was presented. The present study reported the case and discussed the current status of diagnosis of LPLN metastasis of lower rectal cancer and the indication for LPLD. The patient provided written informed consent.

\section{Case report}

The present patient was a 35-year-old female who visited a physician for close examination of fecal occult blood detected at a medical check-up. The patient was diagnosed with rectal cancer following colonoscopy, and was referred and admitted to Tokyo Women's Medical University (Tokyo, Japan). No particular familial or past medical history was known, and no abnormality was detected in blood tests and tumor markers were within the normal ranges (carcinoembryonic antigen, $0.8 \mathrm{ng} / \mathrm{ml}$; cancer antigen 19-9, $6 \mathrm{U} / \mathrm{ml}$ ).

Colonoscopy revealed a superficial elevated-type tumor with a central depression in the lower rectum. This tumor was diagnosed as $\mathrm{T} 1$ deep invasive cancer due to a $\mathrm{V}_{\text {non-structural }}$ pit pattern being observed at high magnification. No features indicating swollen lymph nodes or distant metastasis were present on computed tomography $(\mathrm{CT})$ or $\left[{ }^{18} \mathrm{~F}\right]$-fluorodeoxyglucose

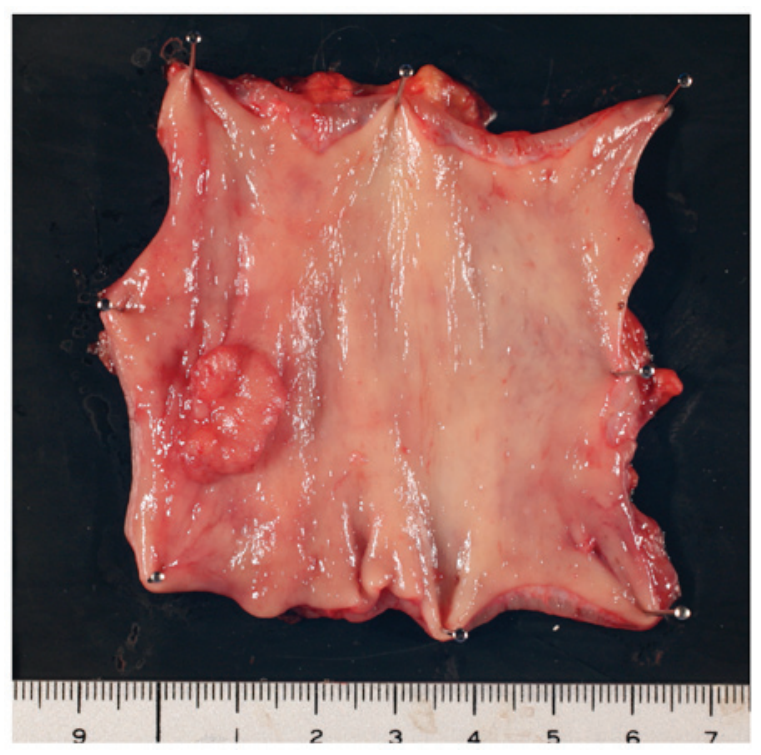

Figure 2. Resection of the tumor revealed that it was the superficial elevated type with a central depression. The size was $16 \times 12 \mathrm{~mm}$.

positron emission tomography $\left({ }^{18} \mathrm{~F}-\mathrm{FDG}\right.$ PET) with CT. However, a swollen lymph node with a short axis of $4 \mathrm{~mm}$ was visualized in the right lateral region on MRI, and this lymph node exhibited a high intensity on DWI, suggesting lymph node metastasis (Fig. 1).

In surgery, low anterior resection and dissection of the regional lymph node and right LPLN were performed. The tumor measured 16×12 mm (Fig. 2), the histopathological grade was 1 , the classification was pT1 and the vertical depth of invasion from the lamina muscularis mucosae 
was 3,000 $\mu \mathrm{m}$ (L1, V0). These findings indicated right LPLN metastasis, as suspected based on the high intensity signal on DWI. No postoperative complications occurred and the patient was discharged 10 days after surgery. Post-operative adjuvant chemotherapy with Tegafur-uracil + leucovorin was performed. The patient remains alive and has been recurrence-free 4 years after surgery.

\section{Discussion}

Diagnosis of lymph node metastasis from rectal cancer is usually made using imaging studies, including endoscopic ultrasound, CT and MRI, which have accuracies of $61-80,56-79$ and $57-85 \%$, respectively (11). The European Society for Medical Oncology guidelines specify MRI as the first-line imaging test as it allows wide evaluation inside and outside the mesorectum (12). MRI has superior contrast resolution in soft tissue and is an excellent method with multiplanar imaging capacity that is generally used for pre-treatment $\mathrm{N}$ staging of rectal cancer (9). The sensitivity and specificity of MRI for diagnosis of lymph node metastasis were revealed to be 77 and $71 \%$, respectively, in a recent meta-analysis (13). However, this meta-analysis also revealed a low diagnostic odds ratio with MRI for rectal cancer lymph node metastasis, indicating that MRI cannot be used to evaluate lymph nodes accurately and is insufficient for complete diagnosis (13).

One of the problems associated with MRI diagnosis of lymph node metastasis is a lack of valid diagnostic criteria. The widely accepted criterion has been the size of lymph nodes on MRI images (14). This is based on the observation that metastasis-positive lymph nodes were larger compared with metastasis-negative nodes. The size criteria, however, has limited value in its performance since small lymph nodes are also often positive and the maximum diameters of positive and negative nodes overlapped to a certain extent on a histogram (14). Morphological imaging criteria, including signal heterogeneity and an irregular border, have also been proposed (14), however, they are not applicable to small lymph nodes sized $\leq 4 \mathrm{~mm}$ (15). Therefore, MRI diagnosis of lymph node metastasis of rectal cancer remains difficult using current techniques.

The utility of DWI for diagnosis of malignant tumors has recently been suggested. When lymph nodes with high signal intensity are detected on DWI, which does not depend on the size, they are considered to be metastasis-positive. For diagnosis of lymph node metastasis of colorectal cancer, Mizukami et al (16) found that the accuracy of DWI of $86.8 \%$ compared favorably with that of $76.0 \%$ for CT. Similarly, Ono et al (6) found an accuracy of DWI of 78.3\%, which was more favorable compared with that of $69.6 \%$ for ${ }^{18} \mathrm{~F}-\mathrm{FDG}-\mathrm{PET}$. DWI also has a higher sensitivity and negative predictive value compared with $\mathrm{CT}$ and ${ }^{18} \mathrm{~F}$-FDG-PET, indicating that fewer cases of lymph node metastasis are diagnosed as false negatives by DWI-MRI. When lymph nodes with high signal intensity are not detected on DWI, these cases are more likely to be negative for lymph node metastasis.

However, differentiation of metastasis-positive and metastasis-negative lymph nodes on DWI is limited since non-metastatic lymph nodes can give high intensity signals in certain cases $(9,17)$. In advanced colorectal cancer, inflamma- tion-induced (reactive) swelling of the lymph nodes is common and may lead to false-positive results (18). By contrast, in early cancer, reactive swelling of lymph nodes does not occur frequently. Choi et al (18) suggested that a high intensity DWI signal for a lymph node in the case of early cancer is more likely to be due to metastasis compared with reactive swelling.

According to the current Japanese guidelines (10), LPLD is recommended for a patient whose tumor has its lower border distal to the peritoneal reflection and the clinical grade of $\geq \mathrm{T} 3$, irrespective of preoperative diagnosis of LPLN metastasis. The recommendation is based on the study in which risk factors for LPLN metastasis were identified to be female gender, tumor location in the lower rectum, histological type of non-well-differentiated adenocarcinoma, maximum tumor diameter $\geq 4 \mathrm{~cm}$ and depth of tumor invasion $\mathrm{T} 3$ or T4, according to the analysis of 1,977 cases accumulated from high volume centers in Japan (5). Analysis of the pre-operative risk factors, the odds ratios for a location in the lower rectum and the depth of tumor invasion T3 and T4 were high, based on which the indication in the current guidelines was established (5). The cohort in the multi-center study, however, was relatively old (1991-1998) and imaging diagnosis of LPLN metastasis was not taken into consideration. The present case was judged to be cT1, which is not included in the indication for LPLD in the current guidelines. Additionally, if the case had been evaluated by CT and ${ }^{18}$ F-FDG-PET/CT only, without MRI, the pre-operative LPLN status would have been judged to be metastasis-negative, and it is unlikely that LPLD would have been performed.

Fujita et al (19) found that CT diagnosis was the most powerful predictor of LPLN metastasis compared with any other independent factors, including pathological status of regional lymph nodes, tumor location and tumor differentiation, suggesting that evaluation of LPLN metastasis by imaging studies is important in determining the treatment strategy for rectal cancer.

In $\mathrm{T} 1$ cases, reactive swelling of lymph nodes is less likely to occur and the incidence of LPLN metastasis is low (0.9\%) (10). Therefore, as with the present case, an LPLN that provides a high signal intensity on DWI in a case of T1 lower rectal cancer is likely to be a metastasis-positive lymph node. DWI appears to be useful for diagnosing or ruling out LPLN metastasis of $\mathrm{T} 1$ lower rectal cancer. A prospective study that examines the role of DWI-MRI on LPLN in planning the treatment strategy for rectal cancer is warranted.

\section{Acknowledgements}

The authors would like to thank Palabra (www.palabra.co.jp) for the English language review.

\section{References}

1. Georgiou P, Tan E, Gouvas N, Antoniou A, Brown G, Nicholls RJ and Tekkis P: Extended lymphadenectomy versus conventional surgery for rectal cancer: A meta-analysis. Lancet Oncol 10: 1053-1062, 2009.

2. Quadros CA, Falcão MF, Carvalho ME, Ladeia PA and Lopes A: Metastases to retroperitoneal or lateral pelvic lymph nodes indicated unfavorable survival and high pelvic recurrence rates in a cohort of 102 patients with low rectal adenocarcinoma. J Surg Oncol 106: 653-658, 2012. 
3. Japanese Society for Cancer of the Colon and Rectum: Japanese classification of colorectal carcinoma. 2nd edition. Tokyo: Kanehara, 2009.

4. Akiyoshi T, Watanabe T, Miyata S, Kotake K, Muto T and Sugihara K; Japanese society for cancer of the colon and rectum: Results of a Japanese nationwide multi-institutional study on lateral pelvic lymph node metastasis in low rectal cancer: Is it regional or distant disease? Ann Surg 255: 1129-1134, 2012.

5. Sugihara K, Kobayashi H, Kato T, Mori T, Mochizuki H, Kameoka S, Shirouzu K and Muto T: Indication and benefit of pelvic sidewall dissection for rectal cancer. Dis Colon Rectum 49: 1663-1672, 2006.

6. Ono K, Ochiai R, Yoshida T, Kitagawa M, Omagari J, Kobayashi H and Yamashita Y: Comparison of diffusion-weighted MRI and 2-[fluorine-18]-fluoro-2-deoxy-D-glucose positron emission tomography (FDG-PET) for detecting primary colorectal cancer and regional lymph node metastases. J Magn Reson Imaging 29: 336-340, 2009.

7. Zhao Q, Liu L, Wang Q, Liang Z and Shi G: Preoperative diagnosis and staging of rectal cancer using diffusion-weighted and water imaging combined with dynamic contrast-enhanced scanning. Oncol Lett 8: 2734-2740, 2014.

8. Kwee TC, Takahara T, Ochiai R, Koh DM, Ohno Y, Nakanishi K, Niwa T, Chenevert TL, Luijten PR and Alavi A: Complementary roles of whole-body diffusion-weighted MRI and 18F-FDG PET: The state of the art and potential applications. J Nucl Med 51: $1549-1558,2010$.

9. Torkzad MR, Påhlman L and Glimelius B: Magnetic resonance imaging (MRI) in rectal cancer: A comprehensive review. Insights Imaging 1: 245-267, 2010.

10. Watanabe $T$, Itabashi $M$, Shimada $Y$, Tanaka $S$, Ito $Y$, Ajioka Y, Hamaguchi T, Hyodo I, Igarashi M, Ishida H, et al: Japanese society for cancer of the colon and rectum (JSCCR) guidelines 2014 for treatment of colorectal cancer. Int J Clin Oncol 20: 207-239, 2015.

11. Klessen C, Rogalla P and Taupitz M: Local staging of rectal cancer: The current role of MRI. Eur Radiol 17: 379-389, 2007.
12. Schmoll HJ, Van Cutsem E, Stein A, Valentini V, Glimelius B, Haustermans K, Nordlinger B, van de Velde CJ, Balmana J, Regula J, et al: ESMO consensus guidelines for management of patients with colon and rectal cancer. A personalized approach to clinical decision making. Ann Oncol 23: 2479-2516, 2012.

13. Al-Sukhni E, Milot L, Fruitman M, Beyene J, Victor JC, Schmocker S, Brown G, McLeod R and Kennedy E: Diagnostic accuracy of MRI for assessment of T category, lymph node metastases and circumferential resection margin involvement in patients with rectal cancer: A systematic review and meta-analysis. Ann Surg Oncol 19: 2212-2223, 2012.

14. Brown G, Richards CJ, Bourne MW, Newcombe RG, Radcliffe AG, Dallimore NS and Williams GT: Morphologic predictors of lymph node status in rectal cancer with use of high-spatial-resolution MR imaging with histopathologic comparison. Radiology 227: 371-377, 2003.

15. Akasu T, Iinuma G, Takawa M, Yamamoto S, Muramatsu $Y$ and Moriyama N: Accuracy of high-resolution magnetic resonance imaging in preoperative staging of rectal cancer. Ann Surg Oncol 16: 2787-2794, 2009

16. Mizukami Y, Ueda S, Mizumoto A, Sasada T, Okumura R, Kohno S and Takabayashi A: Diffusion-weighted magnetic resonance imaging for detecting lymph node metastasis of rectal cancer. World J Surg 35: 895-899, 2011.

17. Heijnen LA, Lambregts DM, Mondal D, Martens MH, Riedl RG, Beets GL and Beets-Tan RG: Diffusion-weighted MR imaging in primary rectal cancer staging demonstrates but does not characterise lymph nodes. Eur Radiol 23: 3354-3360, 2013.

18. Choi J, Oh SN, Yeo DM, Kang WK, Jung CK, Kim SW and Park MY: Computed tomography and magnetic resonance imaging evaluation of lymph node metastasis in early colorectal cancer. World J Gastroenterol 21: 556-562, 2015.

19. Fujita S, Yamamoto S, Akasu T and Moriya Y: Risk factors of lateral pelvic lymph node metastasis in advanced rectal cancer. Int J Colorectal Dis 24: 1085-1090, 2009. 